\title{
Resíduos de frutas transformados em novos produtos alimentícios: uma revisão sistemática
}

\author{
Fruit waste processed into new food products: a systematic \\ review
}

\begin{abstract}
Residuos de frutas transformados en nuevos productos alimenticios: una revisión sistemática
\end{abstract}

Gabriela Arelhano Randolpho ${ }^{1}$ Luane Aparecida do Amaral ${ }^{2}$ Letícia Egidio Arelhano ${ }^{3}$ Elisvânia Freitas dos Santos ${ }^{4}$

${ }^{1}$ Doutoranda do Programa de Pós-Graduação em Biotecnologia na Universidade Federal de Mato Grosso do Sul (UFMS), Campo Grande, MS. E-mail: gabrielaarelhano@yahoo.com.br, ORCID: https://orcid.org/0000-0003-1527-7726

${ }^{2}$ Doutoranda do Programa de Pós-Graduação em Saúde e Desenvolvimento da Região Centro-Oeste na Universidade Federal de Mato Grosso do Sul (UFMS), Campo Grande, MS. E-mail: luapamaral@hotmail.com, ORCID: https://orcid.org/0000-0002-1448-2472

${ }^{3}$ Nutricionista pela Universidade Federal de Mato Grosso do Sul (UFMS), Campo Grande, MS, Brasil. Atualmente, é nutricionista no Hospital de Câncer Alfredo Abrão. E-mail: leticia_arelhano@hotmail.com, ORCID: https://orcid.org/0000-0002-1367-0594

${ }^{4}$ Doutora em Cirurgia Experimental pela Universidade Estadual de Campinas (UNICAMP). Mestre em Alimentos e Nutrição pela Faculdade de Engenharia de Alimentos da UNICAMP. Especialista em Nutrição Humana e Saúde pela Universidade Federal de Lavras (UFLA). Graduada em Nutrição pelo Centro Universitário de Votuporanga. Professora do Curso de Nutrição da Faculdade de Ciências Farmacêuticas, Alimentos e Nutrição (FACFAN) da Universidade

Federal de Mato Grosso do Sul (UFMS), Campo Grande, MS, Brasil. E-mail: elisvania@gmail.com. ORCID: https://orcid.org/0000-0002-1528-6035 
Resumo: O objetivo deste estudo foi descrever o potencial dos resíduos de frutas e sua utilização na formulação de novos produtos alimentícios. Foram utilizadas as bases de dados eletrônicas da SciELO Brasil (Scientific Electronic Library Online) e Science Direct. Foram incluídos artigos completos publicados nos últimos 20 anos, em Português, Espanhol e Inglês. Foram encontrados 515 estudos e selecionados 27, conforme os critérios de inclusão. Os resultados evidenciam que os resíduos de frutas podem ser utilizados para formulação de novos produtos com alto valor nutricional, observa-se elevado teor de fibras e compostos bioativos encontrados nesses resíduos. Dessa forma, o aproveitamento de resíduos de frutas é econômica e nutricionalmente viável e pode ser valioso na formulação de novos produtos.

Palavras-chave: aproveitamento; resíduos; frutas.

Abstract: The objective of this study is to describe the potential of fruit waste and its use in the formulation of new food products. We used the electronic databases of SciELO Brasil (Scientific Electronic Library Online) and Science Direct. We included articles published in the last 20 years in Portuguese, English, and Spanish. We found 515 studies and selected 27 , according to the inclusion criteria. The results show that fruit waste can be used to formulate new products with a high nutritional value, high fiber content, and bioactive compounds found in these residues. Thus, the use of fruit waste is economically and nutritionally viable and can be valuable in the formulation of new products.

Keywords: use; waste; fruit.

Resumen: El objetivo de este estudio fue describir el potencial de los residuos de frutas y su utilización en la formulación de nuevos productos alimenticios. Se utilizaron las bases de datos electrónicas de SCIELO Brasil (Scientific Electronic Library Online) y Science Direct. Artículos completos publicados en los últimos 20 años se incluyeron en Portugués, Español e Inglés. Se encontraron 515 estudios, y se seleccionaron 27, según los criterios de inclusión. Los resultados evidencian que los residuos de frutas pueden ser utilizados para la formulación de nuevos productos con alto valor nutricional, se observa un alto contenido de fibras y compuestos bioactivos encontrados en esos residuos. De esta forma, el aprovechamiento de residuos de frutas es económica y nutricionalmente viable y puede ser valioso en la formulación de nuevos productos.

Palabras clave: aprovechamiento; residuo; frutas. 


\section{INTRODUÇÃO}

A produção estimada de frutas em 2017 foi de, aproximadamente, 44 milhões de toneladas. Esse volume mantém o Brasil como terceiro maior produtor de frutas do mundo, atrás apenas da China e da Índia, respectivamente.

Estima-se que, no mundo, é desperdiçado anualmente 1,3 bilhão de toneladas de alimentos. No Brasil, a quantidade desperdiçada poderia extinguir a fome do país. A conscientização ambiental e uma postura proativa da sociedade atual visam à redução da geração de resíduos, à reutilização de produtos antes do descarte e à reciclagem, como forma de retornar a matéria-prima ao ciclo de produção. Hoje vem crescendo a preocupação com o aproveitamento de resíduos agroindustriais. A caracterização e a avaliação da qualidade dos resíduos industriais são necessárias para incorporação e/ou substituição de novos produtos. Com o conhecimento de seus componentes, sua eficiência no agronegócio é aumentada e seu impacto ambiental reduzido; assim, fatores como uma tecnologia adequada podem ser aplicados e futuras modificações sensoriais podem ser estimadas.

A determinação ou avaliação das características dos resíduos gerados por uma indústria é fundamental, uma vez que o tratamento centralizado deles é desejável e, sempre que aplicável, é uma maneira de economizar em mão de obra e custos de instalações.

Assim, surge a necessidade do aproveitamento de resíduos do processamento de frutas tropicais, sendo uma alternativa para a redução dessas perdas, de forma a contribuir para o desenvolvimento da agroindústria no país.

O tipo de resíduos gerados no processamento de frutas pode ser uma nova fonte alimentar, já que eles têm nutrientes como os antioxidantes, que cada vez mais são utilizados para o retardo do envelhecimento; entretanto o baixo acesso à literatura científica sobre suas possíveis aplicações tem gerado insegurança na aceitação desses subprodutos.

Por meio de uma dieta adequada em quantidade e qualidade, do ponto de vista fisiológico, o organismo adquire a energia e os nutrientes necessários para o bom desempenho de suas funções e para a manutenção de um bom estado de saúde. Os lipídeos e as proteínas constituem um dos 
componentes majoritários dos alimentos, têm alto valor nutritivo e tecnológico. Os carboidratos constituem a principal fonte de energia da dieta e contribuem de forma fundamental no aspecto sensorial dos alimentos. As vitaminas são compostos orgânicos, presentes nos alimentos, essenciais para o funcionamento normal do metabolismo. O organismo não tem condições suficientes de produzi-las nas quantidades necessárias, e a sua falta pode gerar algumas deficiências, sendo preciso ser parte integrante da dieta alimentar humana. A fibra alimentar promove sensação de saciedade e é considerada alimento funcional, pois ajuda no trato gastrointestinal e assegura uma absorção mais lenta dos nutrientes.

Alimentos funcionais, além de suas funções nutricionais básicas, como fornecimento de energia e substrato para a formação de células e tecidos, caracterizam-se por terem em sua composição uma ou mais substâncias capazes de atuar como moduladores dos processos metabólicos, a fim de melhorar as condições de saúde, promover o bem-estar e reduzir o risco de doenças.

Entre os componentes dos alimentos com funcionalidade fisiológica, podemos citar nutrientes, tais como fibras, vitaminas, proteínas; e não nutrientes, como os flavonoides, compostos fenólicos, carotenoides. Os compostos fenólicos encontram-se distribuídos entre as diferentes partes das plantas; porém, sua maior concentração está nas frutas, hortaliças e em seus derivados, são substâncias características de alimentos de origem vegetal. Considerados os antioxidantes de maior ingestão, têm uma vasta gama de atividades biológicas atribuídas a eles como efeito antibacteriano, anti-inflamatório, entre outros.

A importância da fibra alimentar é altamente divulgada nos meios de comunicação, junto da recomendação de seu consumo diário, o que tem levado as indústrias, de certa forma, a buscarem novas fontes de fibras para desenvolver novos produtos e atingir as exigências dos consumidores.

Os resíduos de frutas (RF) derivam principalmente da produção de sucos e polpas, gerando cerca de 30\% a 40\%. Esses resíduos são em maior parte cascas, sementes e caroços. Como esses resíduos produzem danos ao meio ambiente, vários estudos que utilizam os RF na elaboração de novos produtos vêm sendo realizados. 
Nesse sentido, os RF podem ser transformados em farinha ou serem adicionados "in natura", servindo como matéria-prima para novos produtos ricos em nutrientes, de forma a serem ofertados à população alimentos mais saudáveis e com baixo custo. Dessa forma, o objetivo deste estudo foi descrever o potencial dos resíduos de frutas e sua utilização na formulação de novos produtos alimentícios.

\section{MATERIAL E MÉTODOS}

Trata-se de uma revisão sistemática da literatura, focada na questão norteadora: "É viável a utilização de resíduos de frutas na formulação de novos produtos?". Como estratégia na busca de artigos dos últimos 20 anos (janeiro de 1998 a março de 2018), foram utilizadas as seguintes bases de dados: SciELO (Scientific Electronic Library Online) e Science Direct. O fluxograma de seleção está apresentado na figura 1.

As seguintes descrições foram usadas para identificar os artigos: "resíduos industriais AND frutas"; "aproveitamento AND resíduos sólidos"; aproveitamento AND frutas AND análise"; "aproveitamento AND alimento AND resíduo; "cookies AND farinha; "agroindustrial use"; "fruit residue"; "waste products AND fruit"; "industrial waste OR solid waste AND fruit OR waste flour".

Para seleção, foram utilizados os seguintes critérios de inclusão: artigos disponíveis na íntegra em Português, Espanhol ou Inglês. Já os critérios de exclusão foram: resumos e publicações datados anteriormente a 1998 ou que não tinham acesso livre.

Na etapa de elegibilidade, foram lidos os resumos dos artigos e excluídos os trabalhos que não tinha relação com a questão norteadora. Após a leitura total dos artigos, foram incluídos 27 artigos. 
Figura 1 - Fluxograma da seleção de artigos utilizados nesta revisão
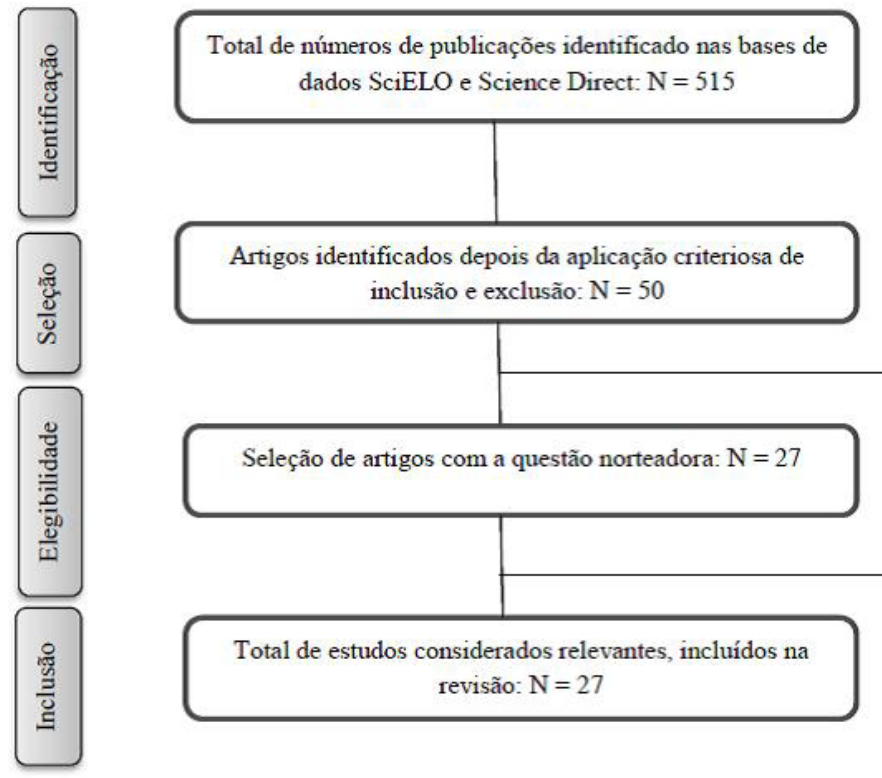

Artigos identificados depois da aplicação criteriosa de inclusão e exclusão: $\mathrm{N}=50$

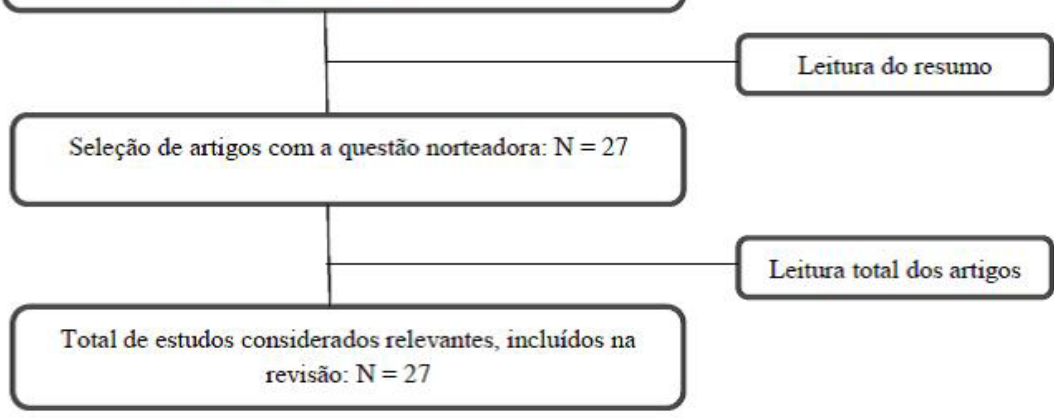

Fonte: Os próprios autores.

\section{RESULTADOS E DISCUSSÃO}

A amostra final desta revisão foi constituída por vinte e sete artigos científicos, selecionados pelos critérios de inclusão previamente estabelecidos. Observa-se que os resíduos encontrados nos estudos foram divididos em: casca $(33,33 \%)$, sementes $(14,81 \%)$, bagaço $(25,93 \%)$ e misto $(25,93 \%)$. As cascas ainda são os principais tipos de resíduos utilizados, porém observa-se que outras partes também estão sendo aplicadas em novos produtos (Tabela 1).

Também pode-se evidenciar o crescimento do número de publicações sobre resíduos nas bases referidas no decorrer dos anos: 2002 (3,70\%); 2005 (3,70\%); 2006 (7,40\%); 2008 (3,70\%); 2011 (7,40\%); 2012 (3,70\%); 2013 (7,40\%); 2014 (22,22\%); 2015 (14,81\%); 2016 (18,51\%). Esses resultados podem ser associados à preocupação com a sustentabilidade no ramo industrial (Tabela 1). 
Tabela 1 - Produtos, análises e resultados principais dos artigos utilizados na revisão

\begin{tabular}{|c|c|c|c|c|}
\hline & Produto Elaborado & Análises & Resultados Principais & Referências \\
\hline & $\begin{array}{l}\text { Produção de enzimas } \\
\text { pectinases a partir de } \\
\text { resíduos de cascas de } \\
\text { laranja. }\end{array}$ & $\begin{array}{l}\text { Físico-químicas } \\
\text { e parâmetros de } \\
\text { otimização para as } \\
\text { enzimas. }\end{array}$ & $\begin{array}{l}\text { A melhor atividade da } \\
\text { pectinase purificada foi em } \\
\text { pH } 7 \text { a } 55 \text { 으, no substrato } \\
\text { de resíduos de cascas de } \\
\text { laranja. }\end{array}$ & $\begin{array}{l}\text { Ahmed et } \\
\text { al., } 2016 \text {. }\end{array}$ \\
\hline & $\begin{array}{l}\text { Formulação de pão com } \\
\text { adição de farinha de } \\
\text { casca de banana. }\end{array}$ & $\begin{array}{l}\text { Físico-químicas e } \\
\text { sensorial. }\end{array}$ & $\begin{array}{l}\text { O biscoito com } 5 \% \text { e o com } \\
10 \% \text { de farinha de casca de } \\
\text { banana foram bem-aceitos. }\end{array}$ & Eshak, 2016. \\
\hline & $\begin{array}{l}\text { Formulação de biscoitos } \\
\text { com farinha de casca de } \\
\text { jabuticaba. }\end{array}$ & $\begin{array}{l}\text { Físico-química, } \\
\text { microbiológicas e } \\
\text { sensorial. }\end{array}$ & $\begin{array}{l}\text { O biscoito com adição } \\
\text { de farinha de casca de } \\
\text { jabuticaba foi bem-aceito. }\end{array}$ & $\begin{array}{l}\text { Zago et al., } \\
2015 .\end{array}$ \\
\hline & $\begin{array}{l}\text { Formulação de biscoitos } \\
\text { com farinha de casca de } \\
\text { goiaba. }\end{array}$ & Físico-química. & $\begin{array}{l}30 \% \text { de farinha de casca } \\
\text { de goiaba em biscoitos foi } \\
\text { aceita em relação a aroma, } \\
\text { sabor e textura. }\end{array}$ & $\begin{array}{l}\text { Bertagnolli } \\
\text { et al., } 2014 .\end{array}$ \\
\hline 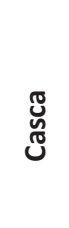 & $\begin{array}{l}\text { Produção de ácido lático } \\
\text { a partir de cascas de } \\
\text { manga. }\end{array}$ & $\begin{array}{l}\text { Planejamento } \\
\text { fatorial. }\end{array}$ & $\begin{array}{l}\text { A produção máxima de } \\
\text { ácido lático foi de } 17.484 \\
\mathrm{~g} / \mathrm{L} \text {, alcançada em } \mathrm{pH} \\
\text { médio inicial de } 10 \text {, seis dias } \\
\text { de incubação e temperatura } \\
\text { de } 35^{\circ} \mathrm{C} \text {. }\end{array}$ & $\begin{array}{l}\text { Jawad et al., } \\
2013 .\end{array}$ \\
\hline & $\begin{array}{l}\text { Formulação de barra } \\
\text { de cereal com casca de } \\
\text { abacaxi. }\end{array}$ & Físico-química. & $\begin{array}{l}\text { Composição centesimal não } \\
\text { variou em função das doses } \\
\text { de radiação utilizadas, } \\
\text { reduzindo apenas os teores } \\
\text { de antioxidantes, fenólicos } \\
\text { e ácidos orgânicos. }\end{array}$ & $\begin{array}{l}\text { Souza et al., } \\
2011 .\end{array}$ \\
\hline & $\begin{array}{l}\text { Formulação de geleia de } \\
\text { casca de cajá-manga. }\end{array}$ & $\begin{array}{l}\text { Físico-química e } \\
\text { sensorial. }\end{array}$ & $\begin{array}{l}\text { Geleia de casca de cajá- } \\
\text { manga foi bem-aceita. }\end{array}$ & $\begin{array}{l}\text { Vanzela et } \\
\text { al., } 2011 .\end{array}$ \\
\hline & $\begin{array}{l}\text { Formulação de compota, } \\
\text { doce, doce glaceado } \\
\text { e geleia das cascas de } \\
\text { melões minimamente } \\
\text { processados. }\end{array}$ & $\begin{array}{l}\text { Físico-químicas e } \\
\text { sensorial. }\end{array}$ & $\begin{array}{l}\text { Boa fonte de fibras e boa } \\
\text { aceitabilidade. }\end{array}$ & $\begin{array}{l}\text { Miguel et } \\
\text { al., } 2008 .\end{array}$ \\
\hline & $\begin{array}{l}\text { Formulação de doce } \\
\text { em calda de cascas de } \\
\text { maracujá-amarelo. }\end{array}$ & $\begin{array}{l}\text { Físico-química e } \\
\text { sensorial. }\end{array}$ & $\begin{array}{l}\text { Doce em calda de cascas de } \\
\text { maracujá-amarelo foi bem- } \\
\text { aceito. }\end{array}$ & $\begin{array}{l}\text { Oliveira et } \\
\text { al., } 2002 .\end{array}$ \\
\hline 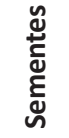 & $\begin{array}{l}\text { Produção de óleo a } \\
\text { partir de sementes de } \\
\text { laranja. }\end{array}$ & Físico-químicas. & $\begin{array}{l}\text { Alta atividade antioxidante } \\
(70,2 \%) \text {. }\end{array}$ & $\begin{array}{l}\text { Jorge, Silva } \\
\text { e Aranha, } \\
2016 .\end{array}$ \\
\hline
\end{tabular}




\begin{tabular}{|c|c|c|c|c|}
\hline & Produto Elaborado & Análises & Resultados Principais & Referências \\
\hline \multirow{3}{*}{ 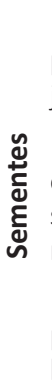 } & $\begin{array}{l}\text { Produção de amido a } \\
\text { partir de sementes de } \\
\text { jaca. }\end{array}$ & Físico-química. & $\begin{array}{l}\text { Teor de amido entre } 92,8 \% \\
\text { e } 94,5 \% \text {. }\end{array}$ & $\begin{array}{l}\text { Madruga et } \\
\text { al., } 2014 .\end{array}$ \\
\hline & $\begin{array}{l}\text { Produção de óleo } \\
\text { extraído a partir de } \\
\text { sementes de laranja, } \\
\text { maracujá e goiaba. }\end{array}$ & Físico-química. & $\begin{array}{l}\text { Podem ser inseridos na } \\
\text { alimentação humana. }\end{array}$ & $\begin{array}{l}\text { Kobori e } \\
\text { Jorge, } 2005\end{array}$ \\
\hline & $\begin{array}{l}\text { Produção de óleos a } \\
\text { partir de sementes de } \\
\text { limão-rosa e siciliano. }\end{array}$ & Físico-química. & $\begin{array}{l}\text { Podem ser inseridos na } \\
\text { alimentação humana. }\end{array}$ & $\begin{array}{l}\text { Reda et al., } \\
2005 .\end{array}$ \\
\hline \multirow{7}{*}{ 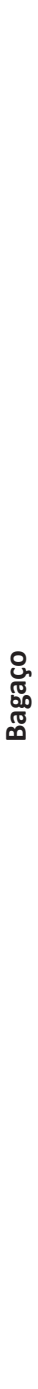 } & $\begin{array}{l}\text { Formulação de biscoito } \\
\text { com farinha de bagaço } \\
\text { de uva. }\end{array}$ & Físico-química. & $\begin{array}{l}\text { O biscoito com adição de } \\
\text { bagaço de uva foi bem- } \\
\text { aceito. }\end{array}$ & $\begin{array}{l}\text { Karnopp et } \\
\text { al., } 2015 .\end{array}$ \\
\hline & $\begin{array}{l}\text { Formulação de biscoito } \\
\text { sem glúten com farinha } \\
\text { de entrecasca de } \\
\text { melancia. }\end{array}$ & Físico-química. & $\begin{array}{l}\text { O biscoito com farinha da } \\
\text { entrecasca de melancia foi } \\
\text { bem-aceito. }\end{array}$ & $\begin{array}{l}\text { Lima et al., } \\
2015 .\end{array}$ \\
\hline & $\begin{array}{l}\text { Produção da farinha } \\
\text { de bagaço de mirtilo } \\
\text { e bagaço de mirtilo } \\
\text { fermentado. }\end{array}$ & Físico-química. & $\begin{array}{l}\text { Fonte de compostos } \\
\text { bioativos. }\end{array}$ & $\begin{array}{l}\text { Goldmeyer, } \\
\text { Penna e } \\
\text { Rosa, } 2014 .\end{array}$ \\
\hline & $\begin{array}{l}\text { Formulação de biscoito } \\
\text { com farinha de bagaço } \\
\text { de uva. }\end{array}$ & Sensorial. & $\begin{array}{l}\text { Biscoitos elaborados com } \\
\text { substituição de até } 50 \% \text { da } \\
\text { farinha de trigo por farinha } \\
\text { integral de aveia e farinha } \\
\text { de bagaço de uva obtiveram } \\
\text { uma boa aceitabilidade. }\end{array}$ & $\begin{array}{l}\text { Piovesana, } \\
\text { Bueno e } \\
\text { Kljn, } 2013 .\end{array}$ \\
\hline & $\begin{array}{l}\text { Produção de doces a } \\
\text { partir do albedo de } \\
\text { maracujá-amarelo } \\
\text { macerado. }\end{array}$ & $\begin{array}{l}\text { Físico-química, } \\
\text { microbiológica e } \\
\text { sensorial. }\end{array}$ & $\begin{array}{l}\text { Doces do albedo de } \\
\text { maracujá-amarelo macerado } \\
\text { foram bem-aceitos. }\end{array}$ & $\begin{array}{l}\text { Dias et al., } \\
2011 .\end{array}$ \\
\hline & $\begin{array}{l}\text { Produção da farinha de } \\
\text { bagaço doce, farinha de } \\
\text { bagaço fermentado e } \\
\text { farinha mista extrusada } \\
\text { de jabuticaba. }\end{array}$ & $\begin{array}{l}\text { Físico-químicas e } \\
\text { sensorial. }\end{array}$ & $\begin{array}{l}\text { Farinha de bagaço de } \\
\text { jabuticaba fermentado } \\
\text { é uma boa fonte de } \\
\text { fibras, constituída de } \\
\text { partículas finas com aroma } \\
\text { característico do fruto e de } \\
\text { sabor azedo-adstringente. }\end{array}$ & $\begin{array}{l}\text { Ascheri, } \\
\text { Ascheri e } \\
\text { Carvalho, } \\
2006 .\end{array}$ \\
\hline & $\begin{array}{l}\text { Produção dos resíduos } \\
\text { de flavedo, albedo e } \\
\text { bagaço de limão. }\end{array}$ & Físico-química. & $\begin{array}{l}\text { Na fração flavedo, foram } \\
\text { determinados os maiores } \\
\text { teores de cinzas e vitamina } \\
\text { C, enquanto o albedo } \\
\text { destacou-se com os maiores } \\
\text { teores de pectina e de fibra } \\
\text { bruta. No bagaço, os teores } \\
\text { de umidade e de proteína } \\
\text { foram os maiores. }\end{array}$ & $\begin{array}{l}\text { Mendonça } \\
\text { et al., } 2006 .\end{array}$ \\
\hline
\end{tabular}




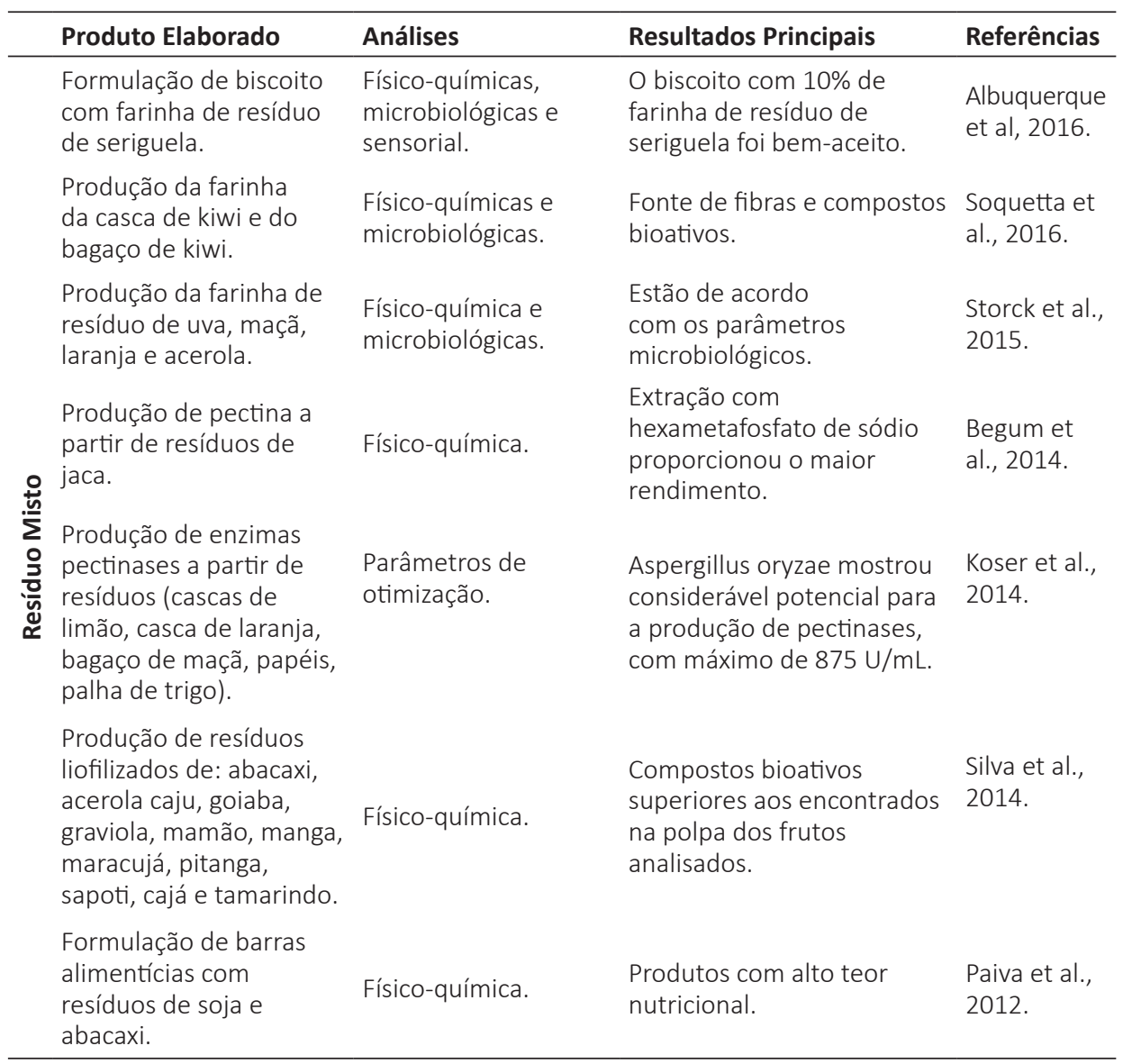

Fonte: Os próprios autores.

Esta revisão encontrou a utilização de resíduos de frutas tanto na elaboração de produtos como liofilizado, farinha, biscoitos, pães e barras alimentares enriquecidos com farinha, geleias e doces, quanto na extração de amido, pectina e enzimas, servindo como matéria-prima para a formulação de novos produtos (Tabela 1).

Os estudos que produziram farinhas a partir de resíduos utilizaram frutos como uva, maçã, laranja e acerola, kiwi, seriguela e jabuticaba (ASCHERI; ASCHERI; CARVALHO, 2006; STORCK et al., 2015; ALBUQUERQUE et al, 2016; SOQUETTA et al., 2016). Segundo Storck et al. (2015), as elaborações de farinhas de resíduo da produção de suco podem ser consumidas, 
enriquecendo ou suplementando preparações alimentícias, de forma a se tornarem uma nova opção para o estudo de diferentes tipos de farinhas e suas propriedades físicas, químicas e sensoriais, possibilitando o aumento das propriedades tecnológicas e funcionais.

Assim, observa-se que novos estudos têm sido descritos na literatura adicionando essas farinhas na elaboração de biscoitos, pães e barras alimentares, produtos amplamente consumidos pela população, de todas as faixas etárias (PIOVESANA; BUENO; KLAJN, 2013; BERTAGNOLLI et al., 2014; KARNOPP et al., 2015; LIMA et al., 2015; ZAGO et al., 2015; ALBUQUERQUE et al, 2016; ESHAK, 2016).

Esses produtos normalmente têm um elevado índice calórico e baixo teor de nutrientes, mas, a partir do momento que são adicionados os subprodutos de frutos, tornam-se mais saudáveis do ponto de vista nutricional, além de trazerem prazer sensorial aos consumidores que, por vezes, não podem consumir o produto padrão, por conter carboidratos simples ou até mesmo glúten (PIOVESANA; BUENO; KLAJN, 2013).

Lima et al. (2015) encontraram em seu estudo que a farinha da entrecasca da melancia é uma alternativa para a indústria de panificação, especialmente com a finalidade de ampliar oferta de alimentos isentos de glúten e com aspectos funcionais. Esse grupo de pesquisadores adicionou este resíduo em biscoitos e obteve um produto de boa aceitação sensorial.

Além disso, Lima et al. (2015) descrevem que a maior barreira encontrada para a utilização de resíduos é o sabor amargo e o escurecimento que, por vezes, esse ingrediente oferece aos produtos. Eles sugerem que tratamentos no material in natura, como o uso de aditivos e/ou outros ingredientes, possam ser formas de otimizar as respostas sensoriais de sabor e textura.

No estudo de Bertagnolli et al., (2014), é possível observar que o percentual de farinha de casca de goiaba influenciou na aceitação do produto: $30 \%$ de farinha de casca de goiaba foi bem-aceita, porém, o percentual de 70\% não obteve boa aceitação, devido à barreira que Lima et al. (2015) relataram em sua pesquisa.

Alimentos que também podem se tornar mais saudáveis são geleias doces e compotas (MIGUEL et al., 2008; DIAS et al., 2011; VANZELA et al., 
2011). Vanzela et al. (2011), ao testarem resíduos de cajá-manga na elaboração de geleia, encontraram maiores teores de proteína, lipídios, cinzas, fibra alimentar, carboidratos totais e pectina, e menores teores de umidade que a polpa, bem como sabor e aroma característicos do fruto e palatabilidade muito semelhante à geleia convencional, sendo um recurso alimentar.

Silva et al. (2014) observaram também que os subprodutos de frutas apresentaram maior conteúdo bioativo do que suas respectivas polpas. Esses compostos têm efeitos benéficos a saúde devido à sua atividade antioxidante, contribuem para o bom funcionamento dos órgãos ou até no combate de doenças. Os estudos analisados constataram altos teores de compostos bioativos $(11,11 \%, n=4)$ e fibras $(14,81 \%, n=4)$ nos RF.

Neste estudo, foi observado que $88,88 \%(n=24)$ dos artigos realizaram análises físico-químicas, 18,51\% ( $n=5)$ microbiológicas, 33,33\% ( $n=$ 9) sensoriais e $3,70 \%(n=1)$ de planejamento fatorial.

A maioria dos alimentos tem uma variabilidade enorme de compostos em sua estrutura, assim, é imprescindível a análise físico-química como forma de caracterização e quantificação deles (SOQUETTA et al., 2016). Já as análises microbiológicas podem monitorar as condições higiênico-sanitárias dos produtos (STORCK et al., 2015). E, ainda, a análise sensorial tem grande importância na avaliação da aceitabilidade ou rejeição de um novo produto (VANZELA et al., 2011).

Além de agregar valor nutricional aos produtos, a utilização de RF evita desperdícios, preservando o meio ambiente, e também é uma alternativa rentável para as indústrias. A partir desta revisão, observa-se que as concentrações de resíduos influenciam significativamente nas propriedades sensoriais e físico-químicas dos produtos elaborados.

Outra forma de reaproveitar resíduos é na extração de compostos como amido, enzimas pectinases, ácido lático e óleos (KOBORI; JORGE, 2005; REDA et al., 2005; JAWAD et al., 2013; KOSER et al., 2014; MADRUGA et al., 2014; AHMED et al., 2016; JORGE; SILVA; ARANHA, 2016). Madruga et al. (2014) utilizaram os RF na obtenção de amido de jaca. O amido é um ingrediente alimentar utilizado como agente geleificante, emulsionante e estabilizante em produtos alimentícios, com efeitos positivos importantes sobre a saúde humana. 
Os óleos de resíduos como laranja, maracujá, goiaba, limão-siciliano e rosa são também alternativas industriais na aromatização de novos produtos e na implementação de ácidos graxos insaturados (KOBORI; JORGE, 2005; REDA et al., 2005; JORGE; SILVA; ARANHA, 2016).

Dessa forma, essas alternativas tecnológicas utilizam o alimento de forma sustentável, reduzem a produção de lixo orgânico, beneficiam a população, promovem reciclagem e solucionam um dos problemas do Brasil em relação à destinação de resíduos sólidos (OLIVEIRA et al., 2002).

\section{CONCLUSÃO}

Em conclusão, os estudos mostram que, nos últimos anos, houve uma grande relevância em pesquisas sobre o uso de resíduos de frutas como matéria-prima no desenvolvimento de novos produtos, pois são ricos em compostos bioativos, antioxidantes, fibras e propriedades funcionais. Tais pesquisas enfatizaram também o aproveitamento de tais resíduos, evitando, consequentemente, desperdícios agroindustriais e preservando o meio ambiente ao mesmo tempo. Portanto, nossa conclusão é de que os RF são viáveis para formulação de novos produtos com alto teor nutricional e baixo custo, e que sua utilização pode ser tanto na forma de liofilizado, farinha, farinha adicionada em biscoitos, pães e barras alimentares, geleias e doces, quanto na extração de amido, pectina, enzimas e ácidos, tornando-se uma alternativa rentável para a indústria.

\section{REFERÊNCIAS}

AHMED, I.; ZIA, M. A.; HUSSAIN, M. A.; AKRAM, Z.; NAVEED, M. T.; NOWROUZI, A. Bioprocessing of citrus waste peel for induced pectinase production by Aspergillus niger; its purification and characterization. Journal of radiation research and applied sciences, v. 9, n. 2, p. 148-54, abr. 2016.

Albuquerque, J. G.; DUARTE, A. M.; CONCEIÇÃO, M. L.; AQUINO, J. S. Integral utilization of seriguela fruit (Spondias purpurea $\mathrm{L}$.) in the production of cookies. Revista Brasileira Fruticultura, Jaboticabal, v. 38, n. 3, e-229, jun./jul. 2016. 
ASCHERI, D. P. R.; ASCHERI, J. L. R.; CARVALHO, C. W. P. Caracterização da farinha de bagaço de jabuticaba e propriedades funcionais dos extrusados. Food Science and Technology, Campinas, v. 26, n. 4, p. 897-905, out./dez. 2006.

BEGUM, R.; AZIZ, M. G.; UDDIN, M. B.; YUSOF, Y. A. Characterization of Jackfruit (Artocarpus heterophyllus) Waste Pectin as Influenced by Various Extraction Conditions. Agriculture and Agricultural Science Procedia, v. 2, p. 244-51, 2014.

BERTAGNOLLI, S. M. M.; SILVEIRA, M. L. R.; FOGA, A. O.; UMANN, L.; PENNA, N. G. Bioactive compounds and acceptance of cookies made with Guava peel flour. Food Science Technology, Campinas, v. 34, n. 2, p. 303-8, abr./jun. 2014.

DIAS, M. V.; FIGUEIREDO, L. P.; VALENTE, W. A.; FERRUA, F. Q.; PEREIRA, P. A. P.; PEREIRA, A. G. T.; BORGES, S. V.; CLEMENTE, P. R. Estudo de variáveis de processamento para produção de doce em massa da casca do maracujá (Passiflora edulis f. flavicarpa). Food Science and Technology, Campinas, v. 31, n. 1, p. 65-71, jan./mar. 2011.

ESHAK, N. S. Sensory evaluation and nutritional value of balady flat bread supplemented with banana peels as a natural source of dietary fiber. Annals of Agricultural Science, v. 61, n. 2, p. 229-35, dez. 2016.

GOLDMEYER, B.; PENNA, N. G.; ROSA, C. S. Características físico-químicas e propriedades funcionais tecnológicas do bagaço de mirtilo fermentado e suas farinhas. Revista Brasileira Fruticultura, Jaboticabal, v. 36, n. 4, p. 980-7, dez. 2014.

JAWAD, A. H.; ALKARKHI, A. F. M.; JASON, O. C.; EASA, A. M.; NORULAINI, N. A. Production of the lactic acid from mango peel waste - Factorial experiment. Journal of King Saud University - Science, v. 25, p. 39-45, jan. 2013.

JORGE, N.; SILVA, A. C.; ARANHA, C. P. M. Antioxidant activity of oils extracted from orange (Citrus sinensis) seeds. Anais da Academia Brasileira de Ciências, v. 88, n. 2, p. 951-8, 2016.

KARNOPP, A. R.; FIGUEROA, A. M.; LOS, P. R.; TELES, J. C.; SIMÕES, D. R. S.; BARANA, A. C.; KUBIAKI, F. T.; OLIVEIRA, J. G. B.; GRANATO, D. Effects of whole-wheat flour and bordeaux grape pomace (Vitis labrusca L.) on the sensory, physicochemical and functional properties of cookies. Food Science and Technology, Campinas, v. 35, n. 4, p. 750-6, out./dez. 2015.

KOBORI, C. N.; JORGE, N. Caracterização dos óleos de algumas sementes de frutas como aproveitamento de resíduos industriais. Ciência e Agrotecnologia, Lavras, v. 29, n 5, p. 1008-14, set./out. 2005. 
KOSER, S.; ANWAR, Z.; IQBA, Z.; ANJUM, A.; AQIL, T.; MEHMOOD, S.; IRSHAD, M. Utilization of Aspergillus oryzae to produce pectin lyase from various agro-industrial residues. Journal of radiation research and applied sciences, n. 3, v. 7, p. 327-32, maio, 2014.

LIMA, J. P.; PORTELA, J. V. F.; MARQUES, L. R.; ALCÂNTARA, M. A.; EL-AOUAR, Â. A. Farinha de entrecasca de melancia em biscoitos sem glúten. Ciência Rural, Santa Maria, v. 45, n. 9, p. 1688-94, set. 2015.

MADRUGA, M. S.; ALBUQUERQUE, F. S. M.; SILVA, I. R. A.; AMARAL, D. S.; MAGNANI, M.; NETO, V. Q. Chemical, morphological and functional properties of Brazilian jackfruit (Artocarpus heterophyllus L.) seeds starch. Food Chemistry, v. 143, p. 440-5, jan. 2014.

MENDONÇA, L. M. V. L.; CONCEIÇÃO, A.; PIEDADE, J.; CARVALHO, V. D.; THEODORO, V. C. A. Caracterização da composição química e do rendimento dos resíduos industriais do limão Tahiti (Citrus latifolia Tanaka). Food Science and Technology, Campinas, v. 26, n. 4, p. 870-4, out./dez. 2006.

MIGUEL, A. C. A.; ALBERTINI, S.; BEGIATO, G. F.; DIAS, J. R. P. S.; SPOTO, M. H. F. Aproveitamento agroindustrial de resíduos sólidos provenientes do melão minimamente processado. Food Science and Technology, Campinas, v. 28, n. 3, p. 733-7, jul./set. 2008.

OLIVEIRA, L. F.; NASCIMENTO, M. R. F.; BORGES, S. V.; RIBEIRO, P. C. N.; RUBACK, V. R. Aproveitamento alternativo da casca do maracujá-amarelo (Passiflora edulis F. FLAVICARPA) para produção de doce em calda. Food Science and Technology, Campinas, v. 22, n. 3, p. 259-62, set./dez. 2002.

PAIVA, A. P.; BARCELOS, M. F. P.; PEREIRA, J. A. R.; FERREIRA, E. B.; CIABOTTI, S. Characterization of food bars manufactured with agroindustrial by-products and waste. Ciência e Agrotecnologia, Lavras, v. 36, n. 3, p. 333-40, maio/ jun. 2012.

PIOVESANA, A.; BUENO, M. M; KLAJN, V. M. Elaboração e aceitabilidade de biscoitos enriquecidos com aveia e farinha de bagaço de uva. Brazilian Journal of Food Technology, Campinas, v. 16, n. 1, p. 68-72, jan./mar. 2013.

REDA, S. Y.; LEAL, E. S.; BATISTA, E. A. C. BARANA, A. C.; SCHNITZEL, E.; CARNEIRO, P. I. B. Caracterização dos óleos das sementes de limão 
rosa (Citrus limonia Osbeck) e limão siciliano (Citrus limon), um resíduo agroindustrial. Food Science and Technology, Campinas, v. 25, n. 4, p. 6726, out./dez. 2005.

SILVA, L. M. R.; FIGUEIREDO, E. A. T.; RICARDO, N. M. P. S.; VIEIRA, I. G. P.; FIGUEIREDO, R. W.; BRASIL, I. M.; GOMES, C. L. Quantification of bioactive compounds in pulps and by-products of tropical fruits from Brazil. Food Chemistry, v. 143, p. 398-404, 2014.

SOQUETTA, M. B.; STEFANELLO, F. S.; HUERTA, K. M.; MONTEIRO, S. S.; ROSA, C. S.; TERRA, N. N. Characterization of physiochemical and microbiological properties, and bioactive compounds, of flour made from the skin and bagasse of kiwi fruit (Actinidia deliciosa). Food Chemistry, v. 199, p. 471-8, 2016.

SOUZA, A. R. M.; SILVA, Y. P. A.; COSTA, N. V.; ALMEIDA, T. L.; ARTHUR, V. LAGE, M. E.; ASQUIERI, E. R.; DAMIANI, C. Irradiação em barras de cereais incorporadas com casca de abacaxi. Pesquisa Agropecuária Tropical, Goiânia, v. 41, n. 4, p. 610-4, out./dez. 2011.

STORCK, C. R.; BASSO, C.; FAVARINI, F. R.; RODRIGUES, A. C. Qualidade microbiológica e composição de farinhas de resíduos da produção de suco de frutas em diferentes granulometrias. Brazilian Journal of food technology, Campinas, v. 18, n. 4, p. 277-84, out./dez. 2015.

VANZELA, E. S. L.; RAMIN, P.; GUEZ, M. A. U.; SANTOS, G. V.; GOMES, E.; SILVA. R. Chemical and sensory characteristics of pulp and peel 'cajá-manga' (Spondias cytherea Sonn.) jelly. Food Science and Technology, Campinas, v. 31, n. 2, p. 398-405, abr./jun. 2011.

ZAGO, M. F. C.; CALIARI, M.; JÚNIOR, M. S. S.; CAMPOS, M. R. H.; BATISTA, J. E. R. Jabuticaba peel in the production of cookies for school food: technological and sensory aspects. Ciência e Agrotecnologia, Lavras, v. 39, n. 6, p. 624-33, nov./dez., 2015. 
